\title{
Das Präludium im deutschsprachigen Raum im 15. und 16. Jahrhundert ${ }^{\star}$
}

\author{
von Kateryna Schöning (Leipzig)
}

Jeder Versuch, das frühe Präludium ${ }^{1}$ als Gattung zu definieren, stößt methodisch rasch an Grenzen, da sich die Begriffe ,Gattung', ,Gattungstyp' und auch ,Stil' auf die Instrumentalmusik des 15. und 16. Jahrhunderts nur schwer anwenden lassen. Das von der Musikwissenschaft vertretene Gattungsverständnis von einem Werktypus, der auf dem Zusammenwirken von spezifisch musikimmanenten und außermusikalischen Kriterien - Stilistik, kompositorische Struktur, ästhetischer Gehalt, Funktion in der Gesellschaft und Kommunikationsstruktur - basiert, ${ }^{2}$ führt bei der Analyse von Präludien der Renaissance in systematischer wie auch in historischer Hinsicht zu Widersprüchen und vermag die Besonderheiten dieser Kunstform nicht zu erklären.

Es ist jedoch nicht sinnvoll, die Existenz der Gattung Präludium vor der Barockzeit gänzlich zu verneinen, denn das Präludium existierte ja seit dem 15. Jahrhundert als eine tradierte Kunstform. Doch stellt sich die Frage, ob es sich hierbei um eine Gattung, eine gattungsstilistische Form oder eine offene stilistische Form handelt. Die Gattungstheorie des 20. Jahrhunderts versteht Gattung als ein System, das in verschiedenen Epochen unterschiedliche Interpretationen erfahren hat. Die Analyse der Instrumentalformen der Renaissance erfordert ein eigenes, historisch adäquates Gattungs- und Stilsystem, bei dem es darum geht, gattungsbestimmende Kriterien festzulegen und Möglichkeiten zu finden, die Vielfalt der Präludien des 15. und 16. Jahrhunderts im geschichtlichen Kontext zu typisieren. Dabei sollte als methodische Grundlage die spezifisch enge Verbindung von Stil, Gattung, Form und Satztechnik im 15. und 16. Jahrhundert nicht außer Acht gelassen werden. ${ }^{3}$ Das Verständnis der Gattungskategorie in dieser Zeit muss also flexible Übergänge zwischen den genannten Kategorien berücksichtigen.

\footnotetext{
* Die vorliegende Studie entstand während eines Forschungsaufenthaltes als Alexander von Humboldt-Stipendiatin an der Hochschule für Musik und Theater „Felix Mendelssohn Bartholdy“ Leipzig. Für Rat und Hilfe bedanke ich mich sehr herzlich bei Prof. Dr. Thomas Schipperges.

${ }^{1}$ Hier und im Folgenden werden die Begriffe ,Präludium', ,Prelude' und ,Präambulum' als Synonyme verwendet, wie es dem ursprünglichen Gebrauch der Termini entspricht. Vgl. hierzu Karin Dietrich, „Praeambulum, praeludium / Prélude, Vorspiel“, in: HMT; Arnfried Edler, Art. „Präludium“, in: MGG2, Sachteil 7, Kassel u. a. 1997, Sp. 17921804; David Ledbetter, Art. "Prelude“, in: NGroveD2, Bd. 20, London 2001, S. 291-293.

2 Zusammengefasst sind hier im Wesentlichen die Hauptthesen der Gattungstheorien von Friedrich Blume, Carl Dahlhaus, Wolfgang Marx und Walter Wiora. Friedrich Blume, „Die musikalische Form und die musikalischen Gattungen", in: Syntagma musicologicum I, hrsg. von Martin Ruhnke, Kassel 1963, S. 480-504; Carl Dahlhaus, "Was ist eine musikalische Gattung", in: Neue Zeitschrift für Musik 135 (1974), S. 620-625; Wolfgang Marx, Klassifikation und Gattungsbegriff in der Musikwissenschaft, Hildesheim 2004; Walter Woira, „Die historische und systematische Betrachtung der musikalischen Gattungen", in: Walter Wiora, Historische und systematische Musikwissenschaft. Gesammelte Schriften, Tutzing 1972, S. 448-476. Die Frage nach einem besonderen Gattungssystem in Instrumentalformen des 16. Jahrhundert wurde von mir bereits kurz berührt in Bezug auf die Fantasie für Laute: Kateryna Shtryfanova, „Kompositorische Strukturen der Lautenfantasie als Gattung in der 1. Hälfte des 16. Jahrhunderts“, in: AfMw 65 (2008), S. 31-44.

${ }^{3}$ Diese methodische These hat Marina N. Lobanova vielfach betont. Marina N. Lobanova, Musical style and genre: history and modernity, Amsterdam 2000 (М. Н. Лобанова, Музыкальный стиль и жанр. История и современность, Москва 1990); М. Н. Лобанова, Западноевропейское музыкальное барокко: проблемь эстетики и поэтики, Москва 1994.
} 
Der vorliegende Aufsatz betrachtet den aktuellen Stand der Gattungsdiskussion, vergleicht die Präludien mit traditionellen Gattungsschemata und nimmt eine Typisierung der Präludien vor. Untersucht werden Orgel- und Lautenpräludien des 15. und 16. Jahrhunderts im deutschsprachigen Raum, insgesamt mehr als hundert Kompositionen. Das Orgelrepertoire umfasst Beispiele von der Mitte des 15. Jahrhunderts bis in die 20er Jahre des 16. Jahrhunderts: ${ }^{4}$

6 Preambula aus der „Tabulatur des Ludolf Bödeker“ (1445)

5 Praeambula von Adam Ileborgh (1448)

Preambulum super g aus der "Orgeltabulatur des Wolfgang de Nova Domo" (vor 1448)

3 Praeambula aus dem Fundamentum organisandi Magistri Conradi Paumann (1452)

3 Prelude aus einer Erlanger Handschrift des 15. Jahrhunderts, D-ERu, Sign. Ms. 554

16 Praeambula aus dem „Buxheimer Orgelbuch“ (1460-1470)

6 Präludien/Praeambula von Hans Kotter (um 1513)

16 Preambala von Leonhard Kleber (1521-1524)

Paeambulum super d aus der „Orgeltabulatur des Fridolin Sicher", St. Galler Orgelbuch, CH-SG 530

Lautenkompositionen entstanden erst später und bilden eine zweite Phase der Entwicklung des Präludiums im deutsch-österreichischen Raum. Diese Periode dauert von den 20er Jahren bis in die 70er Jahre des 16. Jahrhunderts. Folgende Kompositionen wurden in die Untersuchung einbezogen: ${ }^{5}$

5 Priamell von Hans Judenkünig aus Ain schone kunstliche vnderweisung (Wien 1523)

6 Preambeln und ein Preambel oder Fantasey von Hans Newsidler aus Ein newgeordent künstlich Lautenbuch (1536)

3 Preambeln von Hans Newsidler aus Das ander Buch. Ein new künstlich Lautten Buch (1549)

2 Priambeln von Hans Gerle aus Musica teusch auf die Instrument der grossen unnd kleinen Geygen, auch Lautten (Nürnberg 1532)

6 Präludien aus Tabulatur auff die Laudten (Nürnberg 1533)

31 Preambeln von Hans Gerle aus Ein News sehr künstlichs Lautenbuch (Nürnberg 1552)

\footnotetext{
4 Vgl. Martin Staehelin, „Die Orgeltabulatur des Ludolf Bödeker. Eine unbekannte Quelle zur Orgelmusik des mittleren 15. Jahrhunderts", in: Nachrichten der Akademie der Wissenschaften in Göttingen, Philologisch-historische Klasse 5 (1996), S. 157-224; Willi Apel, Keyboard Music of the Fourteenth «) Fifteenth Centuries (= CEKM 1), AIM 1963; Wolfgang Marx, „Die Orgeltabulatur des Wolfgang de Nova Domo“, in: AfMw 55 (1998), S. 152-170; Das Buxheimer Orgelbuch, hrsg. von Bertha Antonia Wallner (= EdM 37-39), Kassel u. a. 1955; Tabulaturen des 16. Jahrhunderts, Teil I: Die Tabulaturen aus dem Besitz des Basler Humanisten Bonifacius Amerbach, hrsg. von Hans Joachim Marx (= SMD 6), Basel 1967; Die Orgeltabulatur des Leonhard Kleber, hrsg. von Karin Berg-Kotterba (= EdM 91-92), Frankfurt a. M. 1987; Tabulaturen des 16. Jahrhunderts, Teil III: Die Orgeltabulatur des Fridolin Sicher, hrsg. von Hans Joachim Marx (= SMD 8), Winterthur 1992.

5 Vgl. Hans Judenkünig, Ain schone kunstliche vnderweisung auff der Lautten vnd Geygen 1523, hrsg. von Helmut Mönkemeyer (= Die Tabulatur 10), Hofheim am Taunus ca. 1969; Hans Newsidler, Ein newgeordent künstlich Lautenbuch 1536, Faks. Stuttgart 2004; Österreichische Lautenmusik im 16. Jahrhundert, bearb. von Adolf Koczirz (= DTÖ XVIII/2 bzw. 37), Wien 1922, Nachdruck Graz 1959; Hans Newsidler, Das ander Buch. Ein new künstlich Lautten Buch 1549, Faks. Stuttgart 1997; Hans Gerle, Ein News sehr künstliches Lautenbuch 1552, Faks. Stuttgart 1997; Documents de musique ancienne, hrsg. von Hélène Charnassé und Raymond Meylan, Ivry s/Seine 1978; Orgeltabulatur des Fridolin Sicher; Lautenmusik aus der Renaissance 2, hrsg. von Adalbert Quadt, Leipzig 1977.
} 
Preambulum aus dem „Lautenbuch des Stephan Craus aus Ebenfurt“ (erste Hälfte des 16. Jahrhunderts)

Präludium von Matthäus Waissel aus Tabulatura continens (1572)

Praeludium aus einer französischen Lautentabulatur (Schreiber D, CH-Bu F. IX. 56)

\section{$I$.}

Eine Darstellung des Präludiums als Gattung ist ein Desiderat der Forschung. Aus einigen wenigen Versuchen, das Präludium im Gattungs- oder Stilkontext darzustellen, resultierten unvollständige und an unterschiedlichen Bedeutungsebenen orientierte Bestimmungen. Oft beziehen sich diese Bestimmungen nur auf einzelne Stücke. „Einen ersten Schritt in Richtung auf stilistische Festigung des Präludiums" ${ }^{\prime \prime}$ sah Willi Apel in Präludien von Wolfgang de Nova Domo und Kompositionen aus dem „Buxheimer

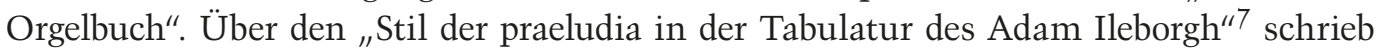
Martin Staehelin. Als „eigenständige Gattung “8 der Orgelmusik wurde das Präludium des 15. Jahrhunderts von Wolfgang Marx beschrieben, der sich auf Werke von Adam Ileborgh und Wolfgang de Nova Domo und auf das Fundamentum organisandi von Konrad Paumann bezog. Den Begriff ,Gattung' benutzte schließlich auch Arnfried Edler. ${ }^{9}$ Die Interpretation der Begriffe ,Gattung' und ,Stil' im Zusammenhang mit dem Präludium ist bei allen genannten Autoren in hohem Maße durch die Methoden der Analyse von Musik späterer Epochen, zum Beispiel der Barockzeit, geprägt.

Nicht zufällig erscheinen in allgemeinen Bestimmungen des frühen Präludiums vorwiegend zwei Aspekte. Einerseits werden Freiheit und Regellosigkeit hervorgehoben und andererseits die Einleitungsfunktion dieser Werke. Ersteres interpretierte Apel als „eine gewisse Freiheit des Stiles, eine Ungebundenheit des Einfalls"10. In diesem Fall überschneidet sich die Bedeutung von ,Präludium' mit jener vieler anderer früher improvisationsnaher Instrumentalformen. ${ }^{11}$ Hingegen sollte es hier doch mehr um Merkmale gehen, die eine Gestalt des Präludiums bedingen. Die Intonations- oder Einleitungsfunktion des Präludiums wird insbesondere von Edler und Karin Dietrich betont. ${ }^{12}$ Dieses Kennzeichen ist zweifellos von großer Bedeutung, denn es bestimmt wichtige Funktionen. Allerdings charakterisiert es das Präludium immer noch nicht ausreichend, sofern man die starke Abhängigkeit der Instrumentalmusik des 15. und 16. Jahrhunderts von der Vokalmusik und die Tatsache, dass auch andere Gattungen der Zeit Einleitungs- oder Vorspielfunktion haben, berücksichtigt. Letzterem wird Hans Heinrich Eggebrecht gerecht, wenn er den Terminus ,Intonation' als funktionalen Oberbegriff für alle diese Formen versteht. ${ }^{13}$

\footnotetext{
${ }^{6}$ Willi Apel, Geschichte der Orgel- und Klaviermusik bis 1700, Kassel u. a. 1967, S. 41.

7 Staehelin, S. 177.

8 Marx, S. 162.

9 Vgl. Arnfried Edler, Geschichte der Klavier- und Orgelmusik, erw. Sonderausgabe Laaber 2007, Bd. 1, S. 534.

10 Apel, Geschichte der Orgel- und Klaviermusik bis 1700, S. 40.

11 ,Freiheit' gehört z. B. zu den am häufigsten genannten Charakteristika der Gattung der Fantasie des 16. Jahrhunderts. Vgl. hierzu Shtryfanova, „Kompositorische Strukturen“.

12 Edler, Geschichte der Klavier- und Orgelmusik; Edler, „Präludium“; Dietrich; Ledbetter.

13 Hans Heinrich Eggebrecht, Studien zur musikalischen Terminologie, Mainz 1955, S. 91.
} 
In den traditionellen Definitionen des Präludiums sind einige Nuancen von entscheidender Bedeutung. So wird die flexible Grenze zwischen dem Präludium als Titel für eine Gattung und als Bezeichnung für einen Entstehungsprozess von Musik hervorgehoben. Beim Präludium handelt es sich um ein eigenständiges schriftlich fixiertes Werk und gleichzeitig um das teilweise notierte Ergebnis einer Improvisationspraxis. Dietrich bemerkt, dass das Wort ,Präludium' als Titel von Musikstücken bereits im 15. Jahrhundert vorkommt, im Sinne einer Gattung hingegen erst im 16. Jahrhundert verwendet wird. Daher begegnet das Präludium als eine eigenständige Werkgattung ihrer Meinung nach erst am Ausgang des 16. Jahrhunderts. ${ }^{14}$ Edler hingegen versteht unter ,Präludium' eine Überschrift für ein Werk der Orgelmusik der ersten Hälfte des 16. Jahrhunderts und ist der Meinung, dass der Begriff erst im 17. Jahrhundert die Bedeutung einer Gattung erhält. ${ }^{15}$ Es zeigt sich also, dass die hier genannten Autoren die Formierung des Präludiums als Gattung nicht ins 15., sondern ins 16. Jahrhundert oder sogar in eine noch spätere Zeit einordnen.

Einige Details der Charakteristik des Präludiums - darunter didaktische Zwecke, die Verbindung von „weltlicher und liturgisch gebundener Musik ${ }^{\prime 16}$ und die Zugehörigkeit ausschließlich zum Bereich der Orgel- oder Lautenmusik bis zum Ende des 16. Jahrhunderts $^{17}$ - werden aus seinen Funktionen erklärt. Es fehlt aber auch hier eine geordnete Darstellung der Informationen. Insbesondere fehlt eine Systematisierung der erhaltenen Quellen aus dem Bereich der Orgel- und Lautenpräludien des 15. und 16. Jahrhunderts.

Zusätzliche Schwierigkeiten bereitet der in der Renaissance verbreitete Usus, Termini und Titel gegeneinander auszutauschen. Abgesehen von den vielen damaligen sprachlichen Varianten konnte der Begriff ,Präludium' nicht nur durch Synonyme, sondern auch durch nicht verwandte und sogar gegensätzliche Termini ersetzt werden. Außer von ,Präludium', ,Preambulum', ,Peambalon', ,Priamel', ,préambule', ,preamble', ,Vor-

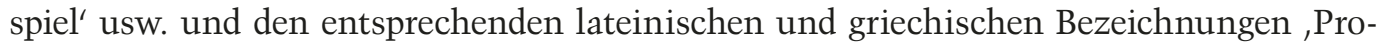
oemium', ,Anabole' und ,Harmonia' wird in der Sekundärliteratur auch von ,Intonatio', ,Ricercar', ,Fantasie', ,Finale' oder ,Nachspiel' gesprochen. ${ }^{18}$ Die beiden letztgenannten Begriffe negieren dabei die dem Präludium zugeschriebene Einleitungsfunktion. Dazu gibt es eine große Menge an Musikstücken, die durch viele Merkmale mit Präludien verbunden sind, aber keinen Titel aufweisen, wie zum Beispiel die Stücke im ersten Teil der Orgeltabulatur von Leonhard Kleber. Die synonymen Benennungen der Präludien beziehen sich auch auf die bisher in diesem Kontext außer Acht gelassene Tradition der Bearbeitungen. ${ }^{19}$

Einige Hinweise in wissenschaftlichen Beiträgen beziehen sich auf die Klassifikation des Präludiums im 15. und 16. Jahrhundert im Hinblick auf die Satztechnik. Hier wird insbesondere der Anteil der Figurationen und der polyphon ausgearbeiteten Partien

\footnotetext{
14 Dietrich, S. 1.

15 Edler, „Präludium", Sp. 1793 f.

16 Dietrich, S. 1.

17 Edler, „Präludium“, Sp. 1792-1794 und Ledbetter, S. $291 \mathrm{f}$.

18 Vgl. etwa Edler, Geschichte der Klavier- und Orgelmusik, S. 528; Klaus Aringer, „Zum Spielvorgang des Beginnens und Schließens in der ältesten Orgelmusik", in: AOl 27 (2001), S. 249-278, hier: S. 254; Dietrich, S. 3.

19 Eine fehlerhafte Benennung der Präludien in heutigen Ausgaben ergibt sich z. B., wenn dasselbe Werk seinerzeit unter verschiedenen Titeln auftrat. So wurde in der Ausgabe Lautenmusik aus der Renaissance 2, S. 4, als Ausgangsbasis für Hans Gerles Priamel (1552) eine Preambel von Antonio Rotta gesehen, in Documents de musique ancienne, S. 60 f., hingegen ein Ricercare (1546) von Antonio Rotta.
} 
eines Werkes angesprochen, doch betrifft dies nur vereinzelte Orgelkompositionen. Apel benennt zum Beispiel einen Ileborgh-Typus, „bei dem über ein paar gehaltenen Tönen frei improvisiert" und "auch mit Akkordfolgen gearbeitet wird" ${ }^{\prime 20}$. Je zwei Typen von Präludien beschreibt Edler für das 15. sowie das 16. Jahrhundert. Im 15. Jahrhundert begegnen uns nach seiner Ansicht Präludien „mit freischweifender Oberstimmfiguration über zwei zu einem Bordunklang verbundenen ausgehaltenen Unterstimmen" und zweistimmige Sätze „mit weniger exzessiver Oberstimmfiguration über den in Minimen und Semiminimen verlaufenden nicht Cantus-firmus-gebundenen Tenor ${ }^{\prime \prime 21}$, im nächsten Jahrhundert ein Typus mit „einer oder zwei melodisch führenden, meist kolorierten Stimmen über dem Gerüst einer von den Unterstimmen gebildeten Konkordanzenfolge" und ein Typus mit „einem mehr polyphonen Wechselspiel zwischen zwei Stimmen ${ }^{\prime 22}$.

II.

Im Hinblick auf die traditionellen Gattungskriterien zeigt sich, dass für die Präludien nur die Kriterien der Existenzweise und Kommunikationsstruktur als bestimmende Kennzeichen Gültigkeit haben. Eines der Merkmale, das die deutsch-österreichischen Präludien verbindet, ist ihre pädagogische Funktion. Seit den frühesten Orgelpräludien aus der Tabulatur des Ludolf Bödeker fungierten die Kompositionen als Übungsstücke. In den Orgelschulen und später dann in den Lautenbüchern geben sie musikalische Anweisungen zum Selbstunterricht. Eine Voraussetzung für solche Übungsstücke war die Orientierung an einem konkreten Instrument - Orgel oder Laute - und an den dafür charakteristischen technischen Problemen in Verbindung mit jeweils aktuellen Spieltraditionen. Das Präludium war also ursprünglich als ein Solostück gedacht, das ganz an der Orgel- oder Lautenspieltechnik ausgerichtet und auf bestimmte methodische Aufgaben begrenzt sein sollte. Obwohl diese Tendenzen nicht bei allen überlieferten Präludien zu beobachten sind, können sie doch als ein wesentliches Kennzeichen dieser Kunstform angesehen werden, das sich auch auf andere Merkmale des Präludiums auswirkt.

Im Bereich der musikimmanenten Kriterien, die eine Gattung bestimmen, spielte das Präludium also eine wichtige Rolle bei der Ausformung und Festigung des instrumentenspezifischen Spielmaterials. Dieses Kriterium gilt aber auch für andere Gattungen wie Intonation, Tokkata, Fantasie und für viele Improvisationsformen, also für einen großen Teil der Instrumentalmusik der Renaissance, und darf deshalb nicht als ein spezifisches Kennzeichen des Präludiums angesehen werden. Auch übernimmt das Präludium in hohem Maße stilistische Eigenarten der Vokal- und Tanzstilistik, die ebenfalls nicht als Charakteristika der Gattung gelten können.

Spezifische Ausprägung erfährt jedoch in Orgel- und Lautenpräludien die modalharmonische Konzeption, was durch den engen Zusammenhang mit der pädagogischen Zielsetzung und mit den Orgel- und Lautenspieltraditionen begründet ist. Viele Orgel-

\footnotetext{
20 Apel, Geschichte der Orgel- und Klaviermusik bis 1700, S. 52.

21 Edler, „Präludium“, Sp. 1793.

22 Ebd., Sp. 1794.
} 
präludien, von den frühesten Präludien aus der Tabulatur des Ludolf Bödeker bis zu den Kompositionen Kotters und Klebers, firmieren unter der Bezeichnung „In Tono ...“ o. ä. Dies verweist auf die liturgische Tradition des Einsingens in einem bestimmten Modus und greift unmittelbar auf die mittelalterlichen Traditionen von Vorspiel oder Intonation zurück. ${ }^{23}$ Die pädagogischen Absichten zeigen sich an dem großen Umfang der verwendeten Modi und an den Vorschriften, die Präludien zu transponieren. Das erstgenannte Phänomen existiert in Orgelpräludien durchgängig seit den frühesten Beispielen. Schon in Präludien aus der Tabulatur des Ludolf Bödeker werden fast alle Töne des Hexachordes eingeübt. So gibt es hier Preambula super $F, g, a, E$ und $d$ (Bödeker57, 91, 96). ${ }^{24}$ Im dritten Teil des Buxheimer Orgelbuches lässt sich ebenfalls eine fünftönige Skala beobachten. Im Unterschied zu Bödekers Tabulatur werden aber die Präludien des Buxheimer Orgelbuchs ausdrücklich in ein Fundamentum integriert und haben Präludien-Varianten im Ton $f$ : Preambula super $C, D, m i$, zweimal super $f$, super sol und Re (Bux232, 233, 234, 235, 240, 241). Im 16. Jahrhundert kreuzt sich diese Linie des Präludiums mit der damals verbreiteten Mode, modal angeordnete Zyklen $\mathrm{zu}$ verfassen. ${ }^{25} \mathrm{Am}$ deutlichsten ist dies bei Kleber ausgeprägt. In seinen Präludien lassen sich drei allerdings nicht komplette Zyklen erkennen: 1. Preambulum (bei Kleber Preambalum oder Preambalon) in ut - re - mi - sol durum - sol molle; 2. in re - fa - fa - sol - sol molle - la - la; 3. in re - sol - la. Die oben erwähnte Tendenz, mit den Präludien auch Übungstücke für Transpositionen zu geben, lässt sich in den Präludien von Ileborgh feststellen. Diese Stücke enthalten entsprechende Hinweise des Komponisten, zum Beispiel Praeambulum super $d, a, f$, et, $g$ oder Sequitur aliud praeambulum super d manualiter et variatur super a $g$ et $c$ (I36, 37 und auch I33). ${ }^{26}$

Im Unterschied $z u$ den Orgelpräludien scheinen die Lautenstücke mehr auf Griffpositionen des Instrumentes hin orientiert zu sein. Die Tradition des In Tono spielt in diesen Werken keine Rolle. Aufgrund der $A$ - oder $G$-Stimmung der Lauten waren die Töne $a$ und $d$ oder $g$ und $c$ am beliebtesten. In fünf Präludien aus Ain schone kunstliche vnderweisung von Judenkünig werden zum Beispiel bei $A$-Stimmung die Töne $d / a, D$, $c / C, D$ und $d$ benutzt. Unabhängig davon, ob ein Orgel- oder ein Lautenstück vorliegt und ob es einen Titel wie In Tono trägt, basiert jedes Präludium auf einem bestimmten modal-harmonischen Konzept und beinhaltet damit auch entsprechende Aufgaben. Das Begreifen des modal-harmonischen Gerüstes und des Prozesses der Ausarbeitung der harmonischen Schemata und Intonationen wird in den Präludien zum wesentlichen Organisationsmittel für sich formende eigenständige Instrumentalkomposition. Es bilden sich für die modal-harmonische Organisation des ganzen Werkes die Schemata

\footnotetext{
23 Edler, Geschichte der Klavier- und Orgelmusik, S. 527 f.

${ }^{24}$ Zur Bezeichnung der Präludien werden im Folgenden Abkürzungen verwendet, die auf Autor oder Quelle und auf die entsprechende Nummer in der Quelle oder Edition hinweisen. Präludien von Ileborgh werden z. B. als I34, I35 usw., das Präludium von Wolfgang de Nova Domo als ND, die Präludien aus der Erlanger Handschrift als Erl59, Erl60 usw., die Präludien aus dem Fundamentum von Paumann als F55, F56 usw., die Werke aus dem Buxheimer Orgelbuch als Bux112, Bux216 usw. und die Präludien von Kotter, Kleber, Newsidler, Judenkünig und Gerle als Kotter32, Kleber6, Gerle1 usw. bezeichnet. Auf Präludien aus der Tabulatur des Ludolf Bödeker wird mit „Bödeker“, der Foliierung nach Staehelin (vgl. Anm. 5) und ggf. dem Titel des Präludiums, auf Präludien von Judenkünig und Newsidler durch Angabe des vollständigen Titels (wie z. B. Judenkünig Das erst Priamell), bzw. bei den vier nicht betitelten Preambeln Newsidlers durch eine Nummerierung in eckigen Klammern (z. B. Newsidler [1.] Preambel) verwiesen.

25 Vgl. hierzu Thomas Schmidt-Beste, Art. „Modus“, in: MGG2, Sachteil 6, Kassel u. a. 1997, Sp. 397-435, hier: Sp. 428.

${ }^{26}$ Vgl. hierzu Edler, Geschichte der Klavier- und Orgelmusik, S. 535 f.
} 
I - V - I, I - III - I, I - IV - I und ihre Varianten heraus. Solche Dispositionen dienen als Grundlage für Moduswechsel, Modi mixti, Polymodalität und erfindungsreiche Akkordformeln. ${ }^{27}$ So kennzeichnet dieses Kriterium das Präludium als eigenständige Kunstform.

Die allgemeine pädagogisch-didaktische Funktion des Präludiums in Abhängigkeit von bestimmten Instrumentalstilen bedingt die Existenz zweier zentraler Typen mit jeweils zwei Untertypen. Der erste Typ umfasst jene Präludien, die von Vokalvorlagen frei sind und vorwiegend primär instrumentaltechnische Aufgaben erfüllen, indem sie instrumentenspezifisches Material ausarbeiten. Hier lassen sich das Präludium als Einleitung und das Präludium als eigenständig entwickelte Instrumentalform unterscheiden. Ein zweiter Typ repräsentiert eine höhere Stufe der Beherrschung des Instrumentalspiels und beinhaltet schwierigere pädagogische Aufgaben; es handelt sich hierbei um Bearbeitungen von Vokal- und Instrumentalvorlagen. Die beiden Untertypen dieses zweiten Typs unterscheiden sich darin, wie stark die Komponisten dem Vorbild folgen: Dem Präludium als genau dem Vorbild folgende Bearbeitung steht das Präludium als variierende Bearbeitung gegenüber.

\section{III.}

Präludien, die von Vokalvorlagen frei und genuin an der Instrumentaltechnik orientiert sind, sind vorwiegend akkordisch-passagenartig gehalten. Diese Kompositionen bilden die größte Gruppe unter den Präludien. Sie stellen eine Anfangsstufe in der Entwicklung des Präludiums dar, die aber im Bereich des Orgel- und Lautenspiels zu unterschiedlichen Zeiten auftritt. Da sich im deutsch-österreichischen Raum die Anfänge eigenständiger Orgelmusik im 15. Jahrhundert, diejenigen eigenständiger Lautenmusik jedoch erst im 16. Jahrhundert beobachten lassen, konzentrieren sich akkordischpassagenartige Orgelpräludien auf das 15. Jahrhundert, während die Lautenpräludien diese Tradition erst im 16. Jahrhundert übernehmen. Zu den erstgenannten rechnen die Stücke aus den Tabulaturen von Ludolf Bödeker, Ileborgh und Wolfgang de Novo Domo sowie Tabulaturen aus der Erlanger Handschrift, aus Paumanns Fundamentum und aus dem Buxheimer Orgelbuch, zu den an zweiter Stelle genannten die Stücke von Hans Judenkünig und Hans Newsidler, also Präludien, die bis zum Jahr 1552 entstanden sind.

Das Präludium als Einleitung artikuliert klar seine charakteristische Funktion. Solche Stücke bereiten intonatorisch oder modal-harmonisch eine nachfolgende Komposition vor; relativ selten (Bux58, 216 und Kleber4) übernehmen sie sowohl in intonatorischer als auch in modal-harmonischer Hinsicht einleitende Aufgaben. Die kompositorische Struktur solcher Präludien stellt eine ein- oder seltener zweiteilige Periode ${ }^{28}$ von oft nur geringem Umfang dar. Hinsichtlich des thematischen Materials unterscheiden die Komponisten streng zwischen passagenartigen und akkordischen Präludien

\footnotetext{
${ }^{27}$ Die modal-harmonische Organisation des frühen Präludiums für Orgel behandelt mein Aufsatz „Über das Verhältnis der modalen und harmonischen Aspekte in deutschen Orgelpräludien des 15. und 16. Jahrhunderts" (im Druck). 28 Der Begriff ,Periode' wird hier in einem erweiterten Sinn verstanden: als ein Gebilde, das nach seiner Funktion und mit den unten beschriebenen Merkmalen eine Vorform der späteren periodischen Strukturen darstellt. Große Ähnlichkeit besteht mit den periodischen Strukturen in der Tanzmusik der Renaissance. Siehe z. B. Edler, Geschichte der Klavier- und Orgelmusik, S. 281.
} 
(Bux216 bzw. 194 [Notenbeispiele 1 und 2]). Dessen ungeachtet ist aber für alle diese Präludien ein akkordisches Gerüst charakteristisch.

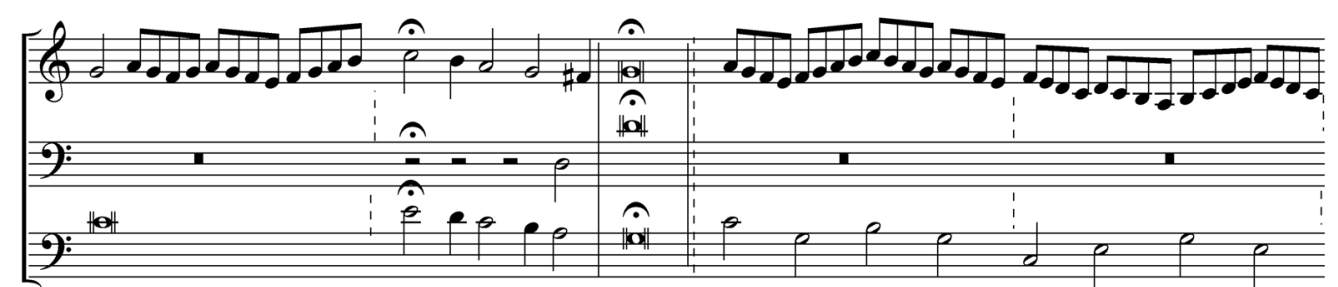

G

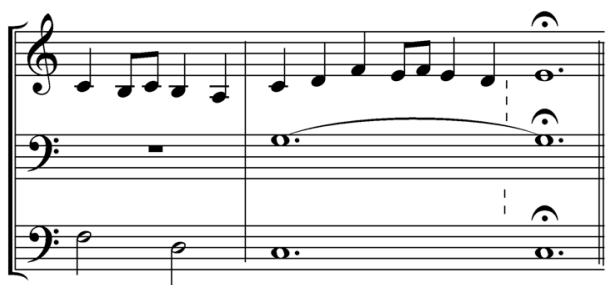

$\mathrm{C}$

Notenbeispiel 1: Buxheimer Orgelbuch, „Praeambulum super C“ (Bux216)

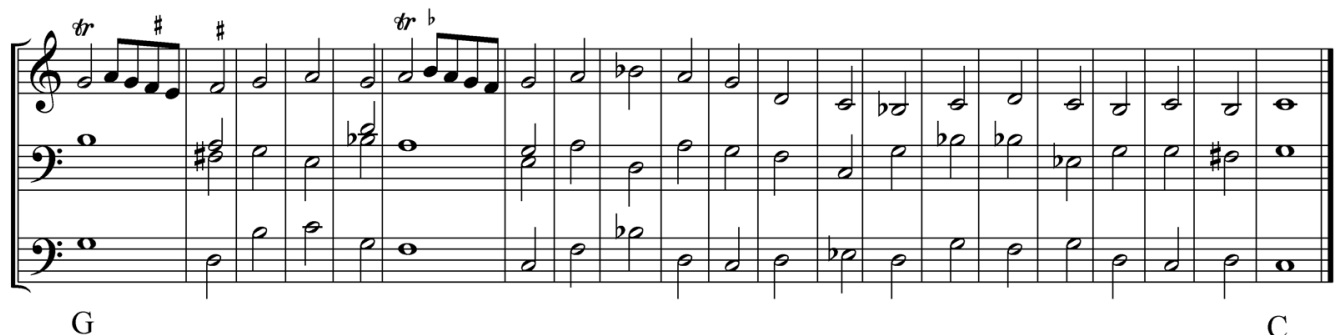

$\mathrm{G}$

Notenbeispiel 2: Buxheimer Orgelbuch, „Praeambulum super C“ (Bux194)

Das Praeambulum Bux216 ist deutlich als zweiteilige einfache Periode angelegt, in der die passagenartigen Figuren am Ende eines jeden Halbsatzes mit einer dreistimmigen Kadenzformel abgeschlossen werden. Trotz der Dominanz der linearen Entfaltung kann hier im Hauptmodus C-Ionisch eine Kadenz-Korrelation G-Ionisch - C-Ionisch nach dem Schema V - I beobachtet werden, die die frei fließende Variabilität der Passagen in der Zweiteiligkeit organisiert. Im Praeambulum Bux194 erfolgt die Suche nach dem Hauptton C ebenfalls von der V. zur I. Stufe, in diesem Fall von G-Ionisch nach CLydisch. Doch geschieht sie ohne Unterteilung in zwei Abschnitte und mit teilweise statisch wirkenden Zusammenklangsfolgen, die häufig $b$ und es mit einbeziehen. ${ }^{29}$ Auf ähnliche Weise konzipierte im 16. Jahrhundert Kleber einige Präludien (akkordisch:

\footnotetext{
29 In den letzten vier Takten dieses Praeambulums wurde von der Herausgeberin Bertha Antonia Wallner in der Oberstimme zweimal anstelle von $h$ ein $b$ vorgeschlagen. In Hinblick auf die offensichtlich vorliegende Parallelkadenz ist das jedoch unmöglich. Ähnlich sieht die Kadenz im Praeambulum super G (Bux53), T. 28-29 aus.
} 
Kleber4; passagenartig: Kleber6, 43, 44, 50). ${ }^{30}$ Eine Weiterentwicklung dieser Tendenz findet man in der Lautenmusik dieser Zeit. Ein eindrucksvolles Beispiel ist das Preambulum aus dem Lautenbuch des Stephan Craus aus Ebenfurt (Notenbeispiel 3).
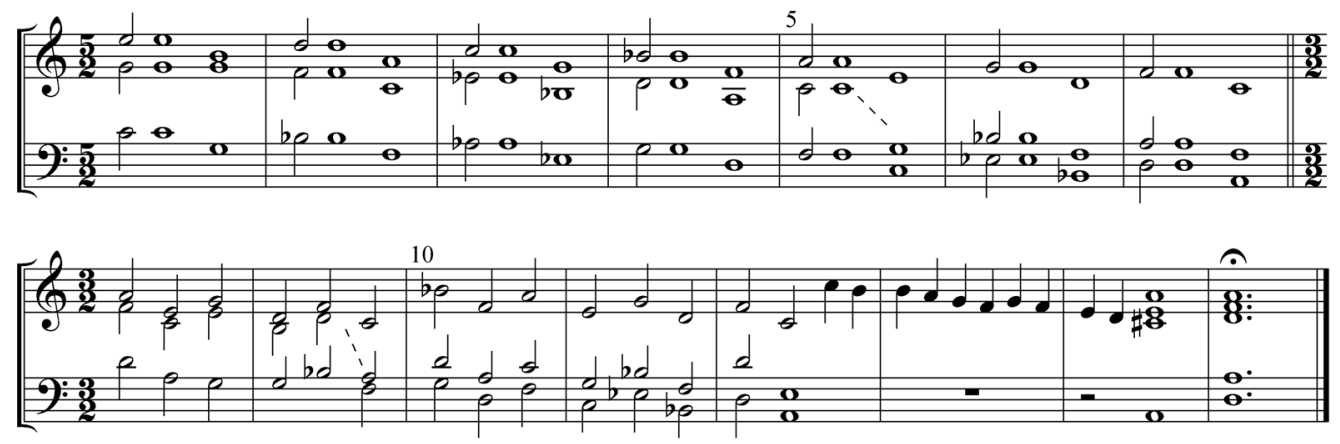

\section{Notenbeispiel 3: Lautenbuch des Stephan Craus aus Ebenfurt, „Preambulum“}

Intensives harmonisches Suchen ist in diesem Stück durch zwei lange akkordische Sequenzfolgen repräsentiert (T. 1-7 und 8-15), die durch ein Gerüst von im Sekundabstand (T. 1-8 und 10-12) bzw. Terzabstand (T. 8-10) aufeinander folgenden absteigenden Quarten organisiert sind und nach einer rein melodischen Passage in eine Kadenz nach $d$ (T. 14-15) münden. Diese Sequenz weist auf das Beherrschen der harmonischen Mittel hin und könnte als weiteres Indiz für die didaktische Funktion des Präludiums verstanden werden. Der Komponist benutzt hier sogar einen Wechsel der metrischen Ordnung zu dreiteiligem Takt, der in ähnlicher Weise sehr oft in der Tanzmusik und in entwickelteren Gattungen wie etwa der Fantasie für Laute des 16. Jahrhunderts gebräuchlich ist. ${ }^{31}$ All dies deutet auf eine eigenständige harmoniebestimmte Instrumentalform des Präludiums hin und spiegelt einen weiteren Schritt in der Entwicklung des instrumentalen Denkens.

Die vertikale Organisation in den akkordischen Abschnitten förderte offensichtlich auch eine strengere rhythmische Konzeption im Vergleich mit den oft nicht gegliederten Passagenabschnitten wie zum Beispiel in Bux216 (Notenbeispiel 1), I33, 34, 37, F55, aber auch im Vergleich mit der Vokalmusik. ${ }^{32}$ Diese Tendenz ist auch im 16. Jahrhundert zu erkennen, wie die Präludien Kleber43 und teilweise Kleber44 demonstrieren. Diese Stücke unterscheiden sich durch ein Akkordgerüst und die harmonische, oft akkordische Fortsetzung von Stücken anderer, ähnlicher Instrumentalgattungen, etwa von Stücken mit dem Titel Finale und Werken ohne Titel. Diese haben zwar Einleitungsfunktion und können wie Präludien kurz und passagenartig sein (Kleber48, 8a, 10a, 45a-c u. a.), jedoch zeigen sie keine modal-harmonische Entfaltung über einer akkordischen Grundlage und sehen eher wie Fragmente einer noch wenig geformten

\footnotetext{
${ }^{30}$ Das allgemeine modal-harmonische Schema kann dabei variiert sein. Außer dem Schema V - I erscheint auch IV - I u. a. Vgl. dazu Shtryfanova, „Über das Verhältnis der modalen und harmonischen Aspekte“.

${ }^{31}$ Metrische Variationen hat z. B. Luis Milán in Fantasien aus El Maestro (1536) benutzt. Vgl. hierzu Shtryfanova, "Kompositorische Strukturen".

32 Siehe z. B. Willi Apel, Die Notation der polyphonen Musik 900-1600, Leipzig 1962, S. 3-18; Edler, Geschichte der Klavier- und Orgelmusik, S. 281. Dies berechtigt dazu, in entsprechenden Passagen die durch senkrechte Striche abgetrennten Einheiten als , Takt' zu bezeichnen.
} 
Improvisation als wie eine werkhafte Gattung aus. Dies könnte in denjenigen Fällen entscheidend sein, in denen es um die Frage geht, ob andere genuine Instrumentalkompositionen, die nicht den Titel Präludium tragen, als Präludien zu betrachten sind. Einige Stücke, wie zum Beispiel in Klebers Tabulatur Cursus Manuale superascendens (Kleber18), bestätigen dabei das Vorherrschen des harmonischen Prinzips in den Präludien.

Präludien als eigenständige entwickelte Instrumentalform sind nicht mit einem nachfolgenden Werk verbunden; sie sind selbstständige Stücke, in denen auf der Basis ihrer modal-harmonischen Pläne eine spezifische Instrumentalfaktur ausgearbeitet und geformt ist. Dabei gelten als charakteristische Kennzeichen dieser Präludien die Formierung des Fakturkontrastes und die Ausarbeitung dieses Kontrastes im Rahmen einer deutlich erkennbaren Form - Merkmale, die die kommunikativen Eigenschaften dieser Gattung nachhaltig verbessern. Berücksichtigt man, dass diese Präludien textlos waren und keine Vokalvorlage hatten, ist dies besonders wichtig.

Passagen- und Akkordabschnitte ${ }^{33}$ werden hier innerhalb eines einzigen Werkes disponiert. Die Abschnitte korrelieren nach den Schemata Akkorde - Passagen (ND, Erl58, Bux210, 206 [Notenbeispiel 4] oder Passagen - Akkorde (Bux195). Schon im 15. Jahrhundert beginnen die Komponisten Akkordabschnitte zu variieren (Bux240, 241, 234). Im 16. Jahrhundert verstärkt sich diese Tendenz (Newsidler, Ein gut Preambel). Gleichzeitig entstehen auf dieser Grundlage die Elemente von Variationen über einem Harmoniegerüst (Kleber68, 1, Kotter33).
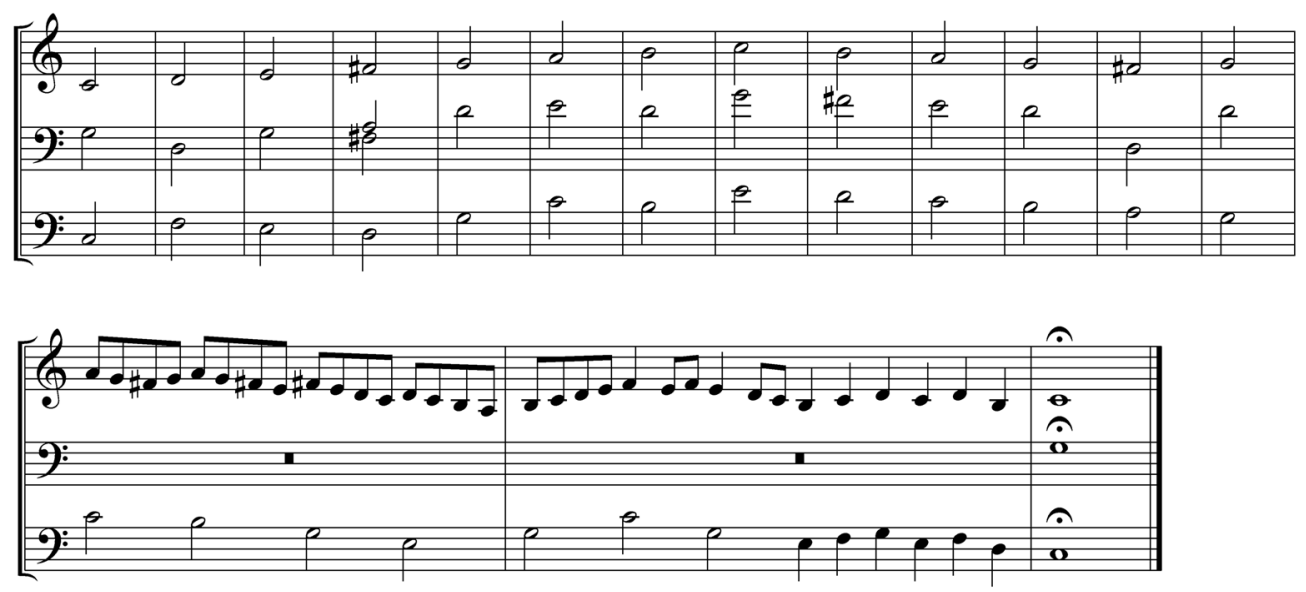

Notenbeispiel 4: „Buxheimer Orgelbuch“, „Sequitur praeambulum super $C$ vel G vel Cc“ (Bux206)

Ein anderes Merkmal der Präludien dieses Typs ist eine Erweiterung des Formenspektrums. Oben gezeigte Beispiele tradieren, wie viele als Einleitungen konzipierte Präludien, eine zweiteilige periodische Struktur. Jedoch erscheinen seit der Erlanger Tabulatur Präludien, in denen einteilige periodische Strukturen eine klare Dreiteiligkeit aufweisen. Dies wird häufig durch einen Fakturkontrast erreicht (Erl59 [Notenbei-

33 Für Präludien des 15. Jahrhunderts ist dieser Begriff noch nicht ohne Einschränkung verwendbar, denn die dort vorliegenden Klangstrukturen basieren auf Intervallzusammenklängen, die eine akkordische Einheit erst formieren. 
spiel 5], 60). ${ }^{34}$ Schon im Verlauf des 15. Jahrhunderts verfestigt sich eine derartige Dreiteiligkeit. Dabei formieren sich die Präludien entweder wie in der Erlanger Tabulatur mit einem Akkordabschnitt in der Mitte (Bux191, 53) oder umgekehrt mit einem mittleren Passagenabschnitt (F55, Bux195). Auch im 16. Jahrhundert bleibt das dreiteilige periodische Schema aktuell (Kleber10).
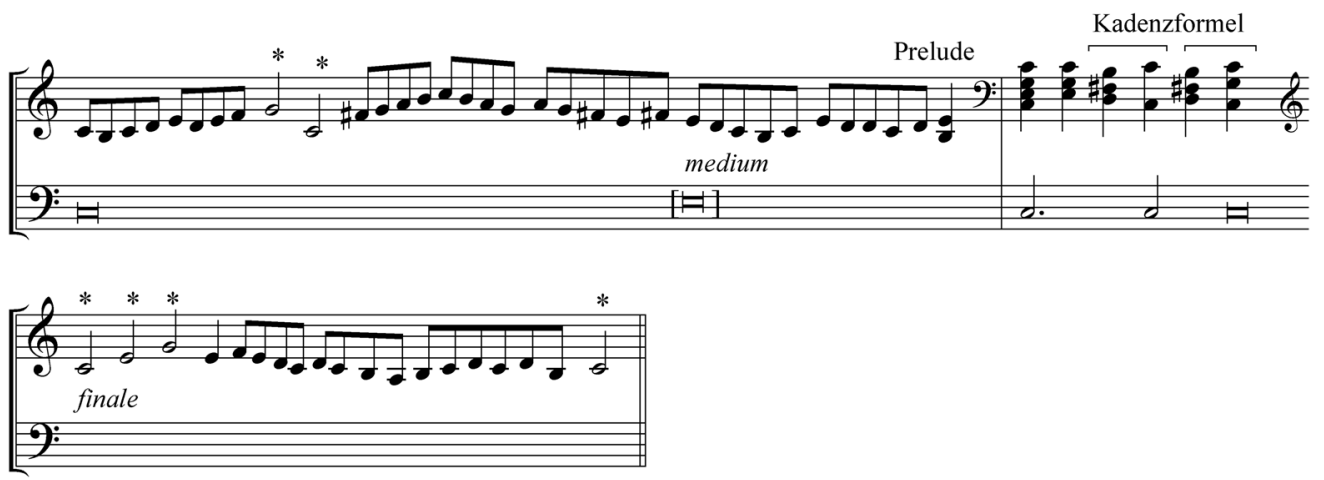

\section{Notenbeispiel 5: Handschrift aus Erlangen, „Prelude“ (Er159)}

Zugleich lassen sich schon innerhalb der Präludien des 15. Jahrhunderts Stücke finden, die sich zu einer einteiligen variierten Form entwickeln und symmetrisch aufgebaut sind (Bux Praeambulum super D, ohne Nummer; Bux232). Im 16. Jahrhundert greifen Newsidler und Kotter diese Anlage auf (Newsidler, Ein seer guter Organistischer Preambel [3.] Preambel, Kotter32). Eine wieder andere formale Lösung finden Komponisten mit zweiteiligen einfachen Formen (Bux233, Kleber53, Jüdenkunig, Das drit Priamel und Das fierd Priamel). Die zur Behandlung des Klangmaterials eingesetzten Mittel sind in diesen Präludien differenzierter als in den einleitenden Präludien, obgleich sich das Augenmerk der Komponisten nach wie vor deutlich auf die Sequenztechnik und den Wechsel zwischen unterschiedlichen metrischen Ordnungen richtet. Als charakteristisches Kennzeichen treten nicht nur akkordische, sondern auch passagenartige Sequenzen auf. Sie kommen vorwiegend in den Mittelabschnitten vor. Dies bezieht sich indes mehr auf die Präludien des 16. Jahrhunderts (Newsidler, alle Präludien; Judenkünig, Das ander Priamel u. a), seltener auf frühere Quellen (Bux53, Praeambulum super $D$, ohne Nummer u. a). Dabei erfolgt der Wechsel von Passagen und Akkorden nun rascher; er verschiebt sich in die Phrasenstrukturen (Newsidler, [1.] Preambel, Ein seer guter Organistischer Preambel). Durch den Wechsel zwischen zwei- und dreiteiliger Taktordnung verstärken die Komponisten die Wirkung des strukturellen und thematischen Kontrastes. Die Abschnitte mit kontrastierender Metrik erscheinen als getrennter Abschnitt entweder in einem mittleren Teil oder am Ende des Werkes (Kleber10, Newsidler, [4.] Preambel).

Ein wesentliches Gestaltungsmittel in den Präludien bildet das Umspielen der abschließenden Wendung, die entweder als harmonisches Kadenzgerüst oder als melodisch-harmonische Kadenzformel ausgebildet ist. Erfüllen derartige Umspielungen im

\footnotetext{
${ }^{34}$ Auf die Dreiteiligkeit beziehen sich auch die Hinweise „medium" und „finale“ in der Quelle.
} 
15. Jahrhundert Kadenzfunktion, so werden sie im 16. Jahrhundert zur Hauptsache (F55, T. 18; F56, T. 5 und 9; F57, T. 8 und 13; Erl59 [Notenbeispiel 5]; Kleberl, T. 1-13; Newsidler, [3.] Preambel). Die Häufigkeit der Verwendung von Kadenzformeln weist womöglich auf ihre außergewöhnliche spieltechnische Funktion hin.

Die Weiterentwicklung der Präludien hinsichtlich der Erweiterung der formalen Anlage und des Beherrschens neuer instrumentaler Techniken bringt in immer größerem Maße eine Verwandtschaft oder Verflechtung der Präludien mit anderen Gattungen mit sich. Am deutlichsten wird dies im 16. Jahrhundert, wenn das Präludium mit anderen Instrumentalgattungen zusammenzuwirken beginnt. Präludien dieser Zeit weisen eine zweiteilige, ausgedehnte Form mit Merkmalen der kontrasthaft-zusammengesetzten Form auf, wobei der zweite Teil wie ein neuer imitatorisch-polyphoner Abschnitt aussieht. Diese Präludien nähern sich in ihrer Form den Fantasien für Laute des 16. Jahrhunderts an. Ein Beispiel gibt Kleber in Preambalum in ut (Kleberl [Schema 1]). Der erste Teil A umspielt die Kadenzformel mit Sequenzen (Material a) und einer Reihe von kurzen variierten Imitationsabschnitten (Material b, c, d und e) und schließt mit der Wiederholung von Material a, wodurch eine klare strukturelle Logik entsteht. Der zweite, kontrastierende Teil ist als ein zweistimmiger variierter Kanon konzipiert. In dieser Weise ausgearbeitete kontrastierende zweiteilige Formen weisen auch die Fantasien für Laute von Luis Milan (Nr. 1, 2, 3), Francesco da Milano (Nr. 39) u. a. auf. ${ }^{35}$ Wie in den Fantasien zeigen sich in solchen Präludien auch erste Ansätze des zukünftigen Zyklus ,Präludium und Fuge', wenn auch noch in einer synkretistischen Einheit. Ein interessantes Beispiel hierfür ist Ein gut Preambel mit fugen aus dem Lautenbuch 1549 von Newsidler. Ein derartiger Titel ist für frühe Präludien singulär. Newsidler baut dieses Stück noch nicht nach dem späteren Muster (improvisatorisch freier Abschnitt imitatorisch-polyphoner Abschnitt) auf, sondern wählt die umgekehrte Reihenfolge: Dem kontrapunktischen Teil, wahrscheinlich der ,fugen', folgt ein kurzer Abschnitt, möglicherweise das ,Preambel', mit passagenartiger Befestigung des Haupttones $a$. Im Ganzen wird dadurch der Kontrast von Akkord- und Passagenfaktur, der für diese Gruppe der Präludien charakteristisch ist, beibehalten.

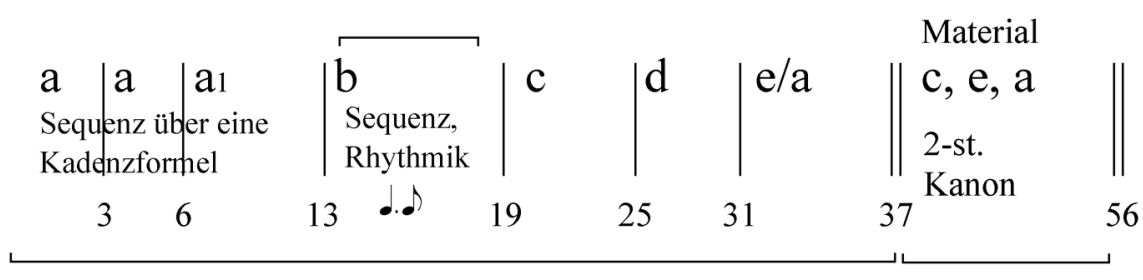

A

B

\section{Schema 1: Kleber, „Preambalum in ut" (Kleber1)}

Die Präludien im 16. Jahrhundert wurden also auch für die Einübung kontrapunktischer Techniken genutzt. Noch im 15. Jahrhundert waren Imitationen den Präludien fremd; der kontrapunktischen Arbeit dienten andere Formen wie etwa Redeuntes aus dem drit-

${ }^{35}$ Vgl. hierzu Shtryfanova, „Kompositorische Strukturen“. 
ten Teil des Buxheimer Orgelbuches. Erst seit der Jahrhundertwende verwendet man in Präludien kurze variierte zwei- und dreistimmige imitatorische Abschnitte, darunter Vorformen fugierter Expositionen (Judenkünig, alle Präludien). Dadurch nähert sich das Präludium dem polyphonen Ricercar und Instrumentalbearbeitungen von Chansons an. Außerdem nehmen die Präludien auch imitatorische Sequenzabschnitte auf, wie sie als formelhafte Abschnitte in Fantasien für Laute vorkommen, etwa kanonische Sequenzabschnitte mit punktiertem Rhythmus (Notenbeispiel 6). ${ }^{36}$

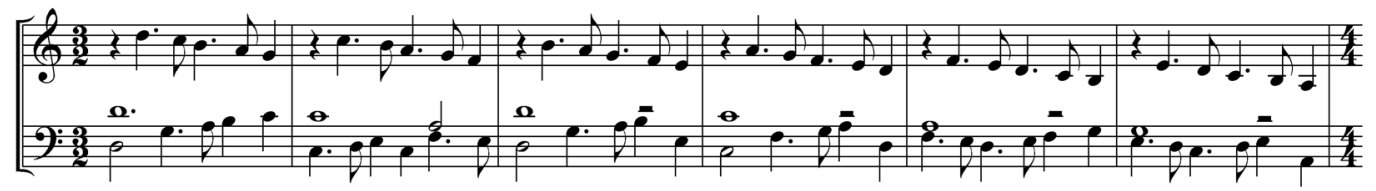

Notenbeispiel 6: Kleber, „Preambalum in ut“ (Kleber1), T. 14-19

Seit Klebers Werken ist dieser Abschnitt mit seinen Varianten in den mittleren Teilen des Präludiums positioniert (Kleberl, T. 14-19; Newsidler, Preambel oder Fantasey, T. 72-77, 105-114, Preambel mit fugen, T. 24-25). Ein imitatorischer Sequenzabschnitt kann, wie in Lautenfantasien dieser Zeit, am Ende des Werkes erscheinen, z. B. in der ersten Fantasie von Valentin Bakfark. Eine Ausnahme lässt sich in Newsidlers Preambel oder Fantasey beobachten. Berücksichtigt man den doppelten Titel sowie den Umfang des Werkes (198 Takte), den mehrschichtigen komplexen Kompositionsbau und die Vielfalt der kontrastierenden Abschnitte, sollte das Werk eher als eine Fantasie angesehen werden.

Im 16. Jahrhundert verfeinert sich die thematische Organisation des Präludiums. Über instrumentale Spielformeln hinaus werden der Vokalmusik entstammende Wendungen eingeführt. Die Komponisten interessieren sich nun für die Bearbeitung dieses vokalen Materials und für die Ausbildung des vokal-instrumentalen thematischen Kontrastes in Präludien. Auf dieser Basis entstehen zwei- und mehrteilige Kompositionen mit getrennten Abschnitten über Instrumental- und Vokalmaterial. Eine symmetrische Form bietet zum Beispiel Newsidler in Ein seer guter Organistischer Preambel (Schema 2).

Thema 1

Thema 2

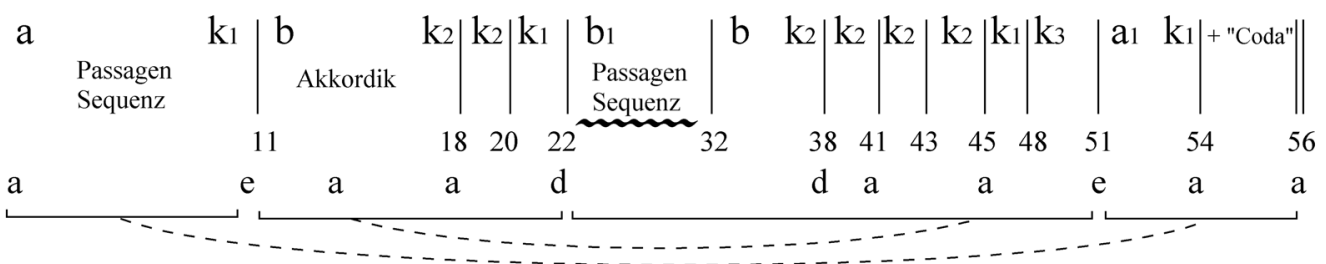

symmetrische Form

Schema 2: Newsidler, „Ein seer guter Organistischer Preambel“

Neben passagenartigem und akkordischem Material (a, Notenbeispiel 7), Umspielung der Kadenzformeln $(\mathrm{k})$ und Akkord- und Passagensequenzen, die auf das 15. Jahrhundert zurückgehen, bilden sich hier kontrastierende Abschnitte über ein schönes Vokal-

${ }^{36}$ Vgl. hierzu ebd. 
thema (b, Notenbeispiel 8). Die große Kunst des Komponisten Newsidler wird deutlich in der überzeugend gestalteten Entfaltung dieser Wendung mit Passagensequenzen in Abschnitt $b_{1}$. Dabei fügt sich die Wendung in die symmetrische Struktur des Werkes ein. Anfangs erscheint sie im Hauptmodus $a$, dann entwickelt es sich im Modus $d_{\text {; }}$ im Reprisenabschnitt kehrt es in den Hauptmodus a zurück. In zweiteiliger Form findet man ein solches Beispiel bei Judenkünig (Das erst Priamell). Insgesamt nähern sich diese Präludien durch derartige motivische und strukturelle Konzeptionen schon sehr der Lautenfantasie jener Zeit an.

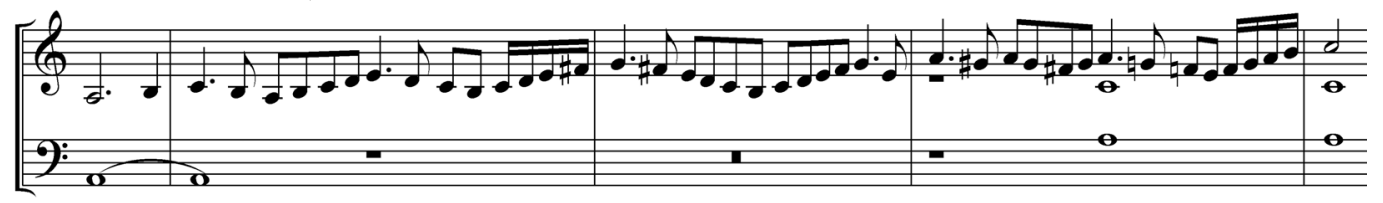

Notenbeispiel 7: Newsidler, Ein seer guter Organistischer Preambel, T. 1-5

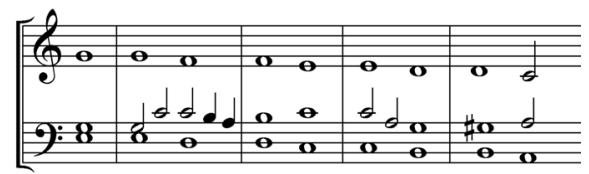

Notenbeispiel 8: Newsidler, Ein seer guter Organistischer Preambel, T. 11-15

Dieser Typ des Präludiums als eigenständige Instrumentalform war also im späteren 15. und im 16. Jahrhundert weit entwickelt. Er unterscheidet sich wesentlich vom Präludium als Einleitung. Dadurch erscheint dieser Präludientyp als Kunstform offener. Dies führt aber zur Auflösung seiner eigenen Merkmale im Zusammenwirken mit Fantasie, Ricercar und den Instrumentalbearbeitungen von Vokalvorlagen. Dadurch erreicht dieser Präludientyp ein anderes Entwicklungsniveau, welches schon große Ähnlichkeit mit dem der Bearbeitungen aufweist.

\section{IV.}

Präludien als Bearbeitungen von Vokal- und Instrumentalstücken etablieren sich erst um die Mitte des 16. Jahrhunderts. Sie bilden eine weitere Etappe in der Entwicklung des frühen Präludiums, die nicht nur mit der Beherrschung der Instrumentalmusik, sondern auch mit verschiedenen Arten des Zusammenwirkens mit anderen Gattungen verbunden ist. Dabei zeigen die Präludien nicht nur allgemein in stilistischer Hinsicht eine Tendenz hin zu Bearbeitungen, sondern formieren einen eigenen Typ, der sich durch bestimmte pädagogische Ziele und Aufgaben definiert. Diese Präludien finden sich vorwiegend in der Lautenmusik der zweiten Jahrhunderthälfte.

Das Präludium als eine genau dem Vorbild folgende Bearbeitung entstand aus dem pädagogischen Bestreben, Originalwerke des Lautenrepertoires bekannter Komponisten unverändert wiederzugeben. Charakteristische Beispiele hierfür stellen die Präludien von Gerle dar. In seinen 31 Präludien aus Ein Newes sehr künstlichs Lautenbuch (1552) verfolgt der Komponist das Ziel, Werke von italienischen Meistern der 30er und 40er 
Jahre des 16. Jahrhunderts in die deutsche Lautentabulatur zu übertragen und auf diese Weise im deutschsprachigen Raum zu verbreiten. ${ }^{37}$ Erwähnenswert ist, dass Gerle die Komponisten nennt, deren Werke er übertragen hat, zum Beispiel Das 1. Preambel Joan. Maria oder Das 12. Preambel Simon Gintzler u. a. ${ }^{38}$ Als Vorlagen für die Übertragungen wurden Ricercare und Fantasien ausgewählt, also zwei verbreitete Instrumentalgattungen des 16. Jahrhunderts. Alle Übertragungen sind aber in „Preambel“ umbenannt worden. ${ }^{39}$

Bei dem Versuch, mit den Übertragungen möglichst nahe am Original zu bleiben, wurde die Aufmerksamkeit für die Originalquellen erhöht. Gerle bemühte sich vor allem darum, die Imitationen möglichst genau wiederzugeben. Dies bezieht sich insbesondere auf die Anfangsabschnitte (Crema, Recercar segondo und Gerle2, T. 1-4 [Notenbeispiel 9]). Die in den italienischen Tabulaturen durch Wiederholungszeichen angezeigten Wiederholungen schrieb Gerle aus; er schrieb auch einige im Original nicht wiederholte Abschnitte doppelt (Crema, Recercar quarto und Gerle3, T. 37-39, 40-43). Alle vermeintlichen Fehler der Vorlagen wurden pädagogisch sorgfältig korrigiert. Bei der Übertragung der Ricercare von Giovanni Maria da Crema aus der Intabolatura de lauto. Libro Primo (1546) verschob Gerle die im Original möglicherweise ,fehlerhaften' unregelmäßig geschriebenen Taktstriche (Crema, Recercar primo und Gerle1, T. 2-3 [Notenbeispiel 10]; Crema, Recercar sexto und Gerle4, T. 23-24). In einigen Fällen fügte er im Original fehlende Noten ein. Er griff jedoch auch anderweitig ein, indem er Figurationen oder vereinfachte Passagen verkomplizierte (Crema, Recercar undecimo und Gerle6, T. 25-27 [Notenbeispiel 11]; Crema, Recercar decimoquinto und Gerle8, T. 1 [Notenbeispiel 12]; Crema, Recercar quarto und Gerle3, T. 23), fünfstimmige Akkorde zu vierstimmigen umformte und kurze figurierte Formeln einfügte (Crema, Recercar decimo und Gerle7, T. 58-59; Crema, Recercar undecimo und Gerle6, T. 3). In Gerles Das 2. Preambel ergibt sich gegenüber Cremas Recercar segondo auch eine Uminterpretation des Modus, indem Gerle durch das as in T. 2 eine phrygische Komponente hineinbringt [Notenbeispiel 9]. Ansonsten finden sich keine prinzipiellen Unterschiede zwischen den Originalwerken und ihren Übertragungen. ${ }^{40}$

\footnotetext{
${ }^{37}$ Hierzu Mirko Arnone, Art. „Gerle Hans“, in: MGG2, Personenteil 7, Kassel u. a. 2002, Sp. 788-790.

38 Siehe Hans Gerle, Ein News sehr künstliches Lautenbuch 1552, Hans Newsidler, Das ander Buch 1549, Hans Newsidler, Das dritt Buch 1544.

${ }^{39}$ Es wurden von Gerle die Recercare von Giovanni Maria da Crema (1546), Dominico Bianchini detto Rossetto (1546), Simon Gintzler (1547) und Antonio Rotta (1546) sowie Fantasien von Francesco da Milano (1536), Pietro Paolo Borrono di Milano (1536), Marco d'Aquilla (1536), Alberto da Rippe (1536) und Johannes Jacobo Albutio di Milano (1536) übertragen.

40 Es lassen sich nur geringe Abweichungen von Original bemerken, die keine offensichtlichen Gründe haben, z. B. eine Vereinfachung der Rhythmik (Crema, Recercar decimoquarto und Gerle5, T. 21; Crema, Recercar quarto und Gerle3, T. 2 und 34).
} 

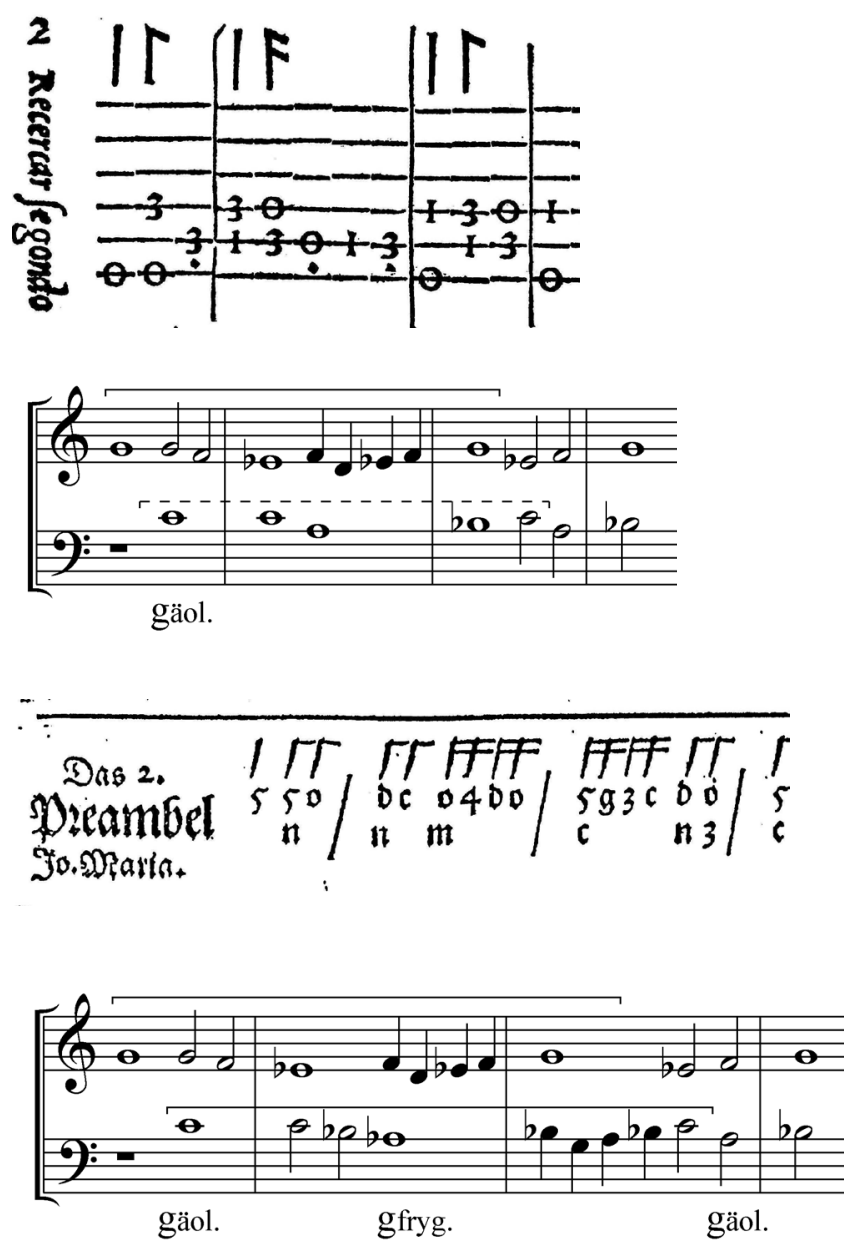

Notenbeispiel 9: Crema, „Recercar segondo“, T. 1-4 und Gerle, „Das 2. Preambel“ (Gerle 2), T. 1-4 

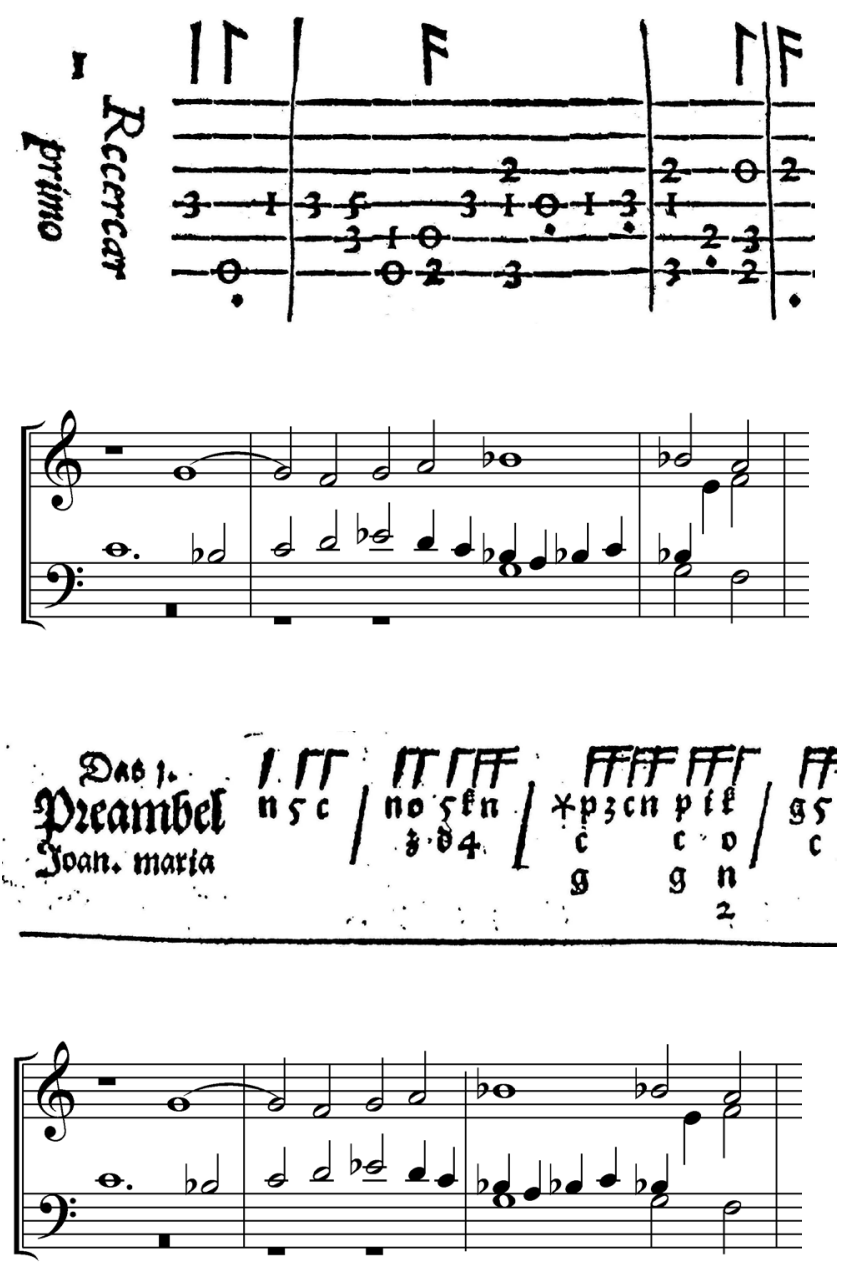

Notenbeispiel 10: Crema, „Recercar primo“, T. 1-3 und Gerle, „Das 1. Preambel“ (Gerle 1), T. 1-3 

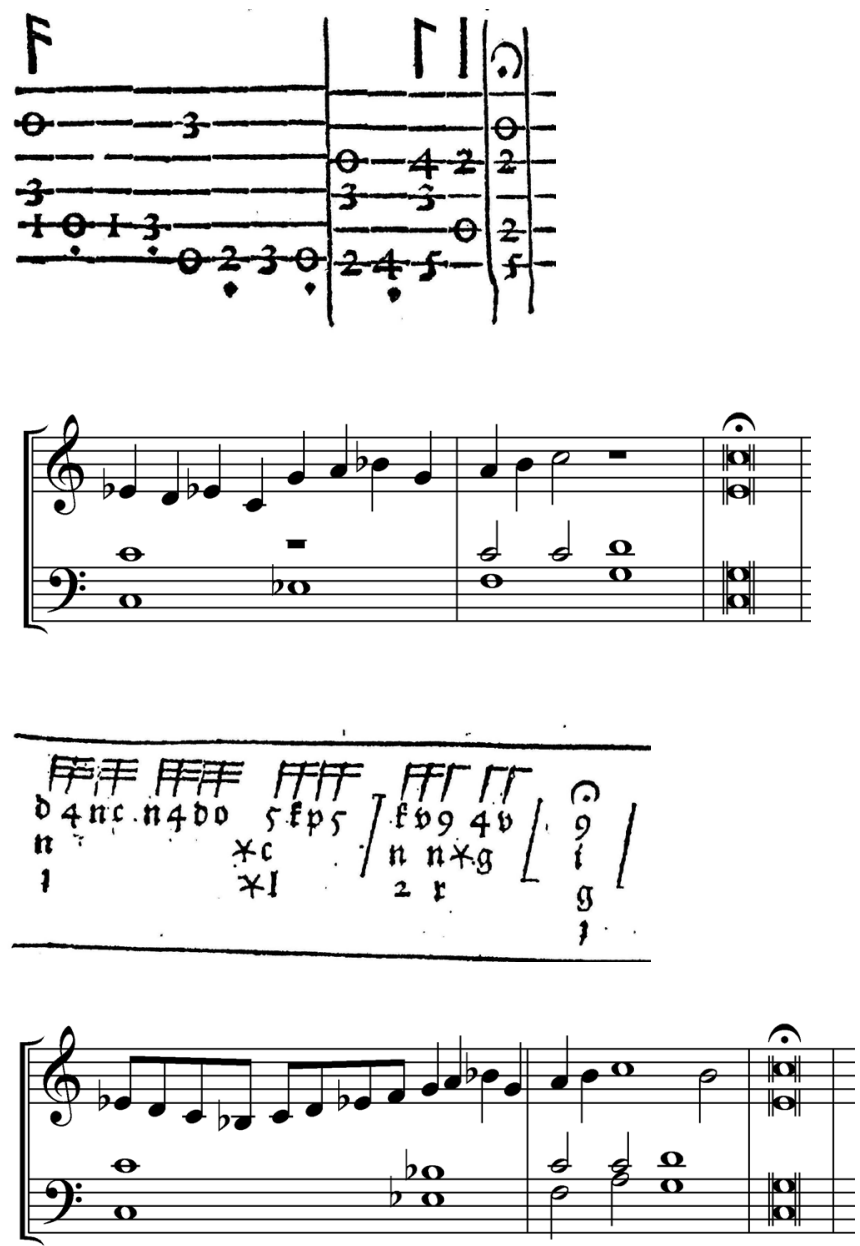

Notenbeispiel 11: Crema, „Recercar undecimo“, T. 25-27 und Gerle, „Das 6. Preambel“ (Gerle 6), T. 25-27 

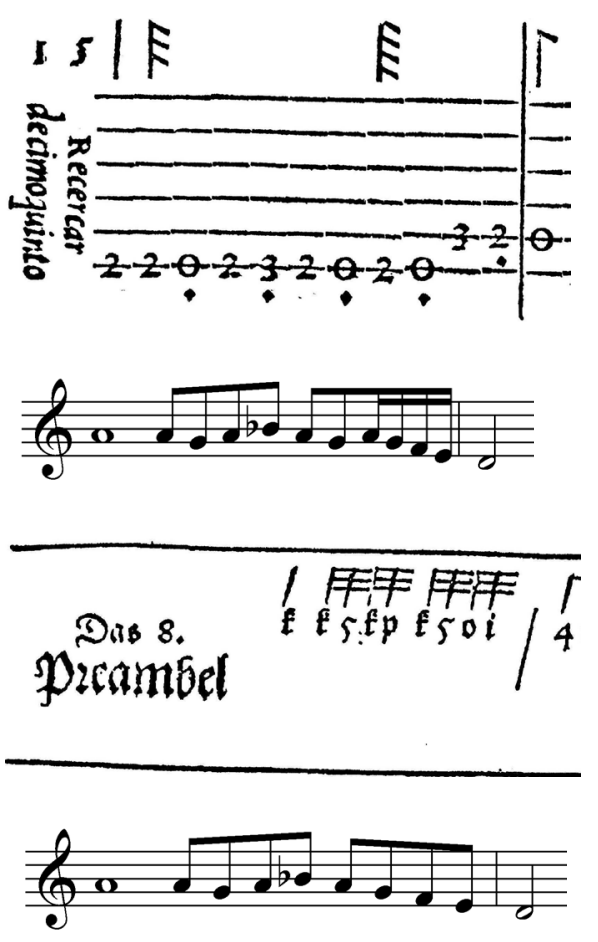

Notenbeispiel 12: Crema, „Recercar decimoquinto“, T. 1 und Gerle, „Das 8. Preambel“ (Gerle 8), T. 1

Besonderes pädagogisches Interesse riefen offensichtlich die schwierigen polyphon-imitatorischen Ricercare und Fantasien hervor. Unter den transkribierten Ricercaren etwa begegnet man am häufigsten einem Ricercartyp mit zwei Soggetti ${ }^{41}$ in einer zweiteiligen variierenden Form; er basiert durchgängig auf kontrapunktischer Satzweise, wobei sich fugierte drei- und vierstimmige Expositionen (Gerle1, 2, 5, 6, 8, 10, 12), Strettaund Kanon-Technik (Gerle2, 4, 5) sowie Intermedien (Gerle2) beobachten lassen. In den Präludien spiegelt sich auch die Tendenz der Ricercare hin zu kontrasthaft-zusammengesetzter Bauweise mit mehreren Soggetti wider (Gerle3, 13, 18); hier sind die Verstärkung des Kontrastes zwischen den Soggetti (Gerle8, 12, 14) und die Gestaltung von sich davon abhebenden Abschnitten mit Passagen oder harmonischen Sequenzen (Gerle7, $10,12,14,16)$ von großer Bedeutung. Eine Parallele haben diese Präludien in Ricercaren mit zwei Soggetti, die in eine dreiteilige Form mit kontrastierendem Mittelabschnitt eingebaut sind (Gerle15).

Unter jenen Präludien, die ihre Vorlage exakt transkribieren, gibt es auch einige singuläre Erscheinungen. Dazu gehört das Praeludium in CH-Bu F. IX. 56. Das Stück stellt

\footnotetext{
${ }^{41}$ Obgleich sich im 16. Jahrhundert in der Instrumentalmusik ein gleitender Übergang zwischen jenen Gebilden, die eindeutig als ,Themen' bezeichnet werden können, und solchen Gebilden, die unter dem Begriff ,Soggetti' gefasst werden sollten, findet, möchte ich folgende Unterscheidung treffen: ,Soggetti' sind nur diejenigen Wendungen, die weniger motivisch geprägt und bei ihrer Wiederkehr schlecht erkennbar sind. In Ricercaren sind die Soggetti oft zu Themen geworden.
} 
einen geradtaktigen Tanz dar, der vermutlich das - uns nicht überlieferte - Original unverändert wiedergibt [Notenbeispiel 13]. Eine zwei- und dreistimmige Kanonübung ist die Grundlage für das Preambalon in fa aus der Tabulatur von Kleber (Kleber47 [Notenbeispiel 14]). Auf die Besonderheit dieses Präludiums soll möglicherweise die Nennung eines Namens im Titel - Magister Jörg Schapf - hinweisen.

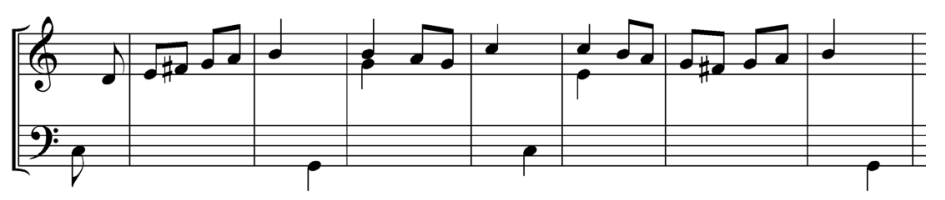

Notenbeispiel 13: Anonymus, „Praeludium“, T. 1-8

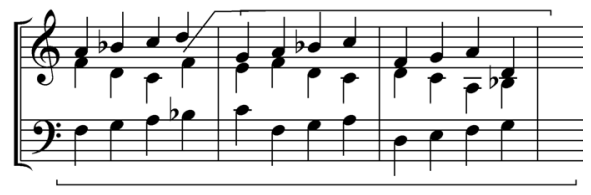

Notenbeispiel 14: Kleber, „Preambalon in fa“ (Kleber47), T. 1-3

Das Präludium als variierende Bearbeitung ist schwer zu definieren. Die zu diesem Typus gehörenden Präludien sind Kompositionen, an denen die Tendenz des 16. Jahrhunderts zu Bearbeitungen zu erkennen ist. Ihre spezifischen Merkmale sind davon kaum zu trennen. Außerdem nähern sich diese Präludien aber genuinen Instrumentalpräludien, die durch ihre Offenheit gegenüber anderen Gattungen wie etwa Fantasie und Ricercar gekennzeichnet sind. Das Präludium als variierende Bearbeitung kann jedoch nicht nur als eigenständiger Typ bestimmt werden, sondern in ihm sind auch auf das 15. Jahrhundert zurückgehende Traditionen zu finden.

Frühe Vertreter für das Präludium als variierende Bearbeitung können in Orgelpräludien von Ileborgh, in Stücken aus der Erlanger Handschrift und teilweise in Paumanns Fundamentum gesehen werden (I33, 36; Er158, 60; F56, 57). Einerseits sind diese Stücke, wie oben beschrieben, dem Typ des genuinen Instrumentalpräludiums zuzuordnen, denn sie sind als ein- oder zweiteilige Periode mit passagenartigem oder akkordischem Material und mit ausgearbeiteten modal-harmonischen Kompositionsplänen gestaltet. Andererseits verweist aber die Faktur dieser Präludien darauf, dass sie als Tenorbearbeitung anzusehen sind. Diese Präludien stellen also Zwischenformen dar und demonstrieren den engen Zusammenhang zwischen Bearbeitung und Präludium als eigenständiges Werk. Doch hoben sich diese Präludienbearbeitungen schon im Laufe des 15. Jahrhunderts von anderen Präludien deutlich ab. Sie entstanden allerdings nur in geringer Anzahl, bildeten also eher die Ausnahme, ungeachtet dessen, dass Tenorbearbeitungen eine wichtige Grundlage der frühen Orgelstücke und insbesondere der Fundamenta waren. Als Cantus-firmus-Variation gestaltete Präludien finden wir vereinzelt im dritten Teil des Buxheimer Orgelbuchs (Bux232). 
Im 16. Jahrhundert entwickelt sich ein Interesse an Präludien, die ein Vokal- oder Tanzthema übernehmen. Es gibt nicht sehr viele Beispiele, doch sind diese sehr prägnant. So lässt sich eine Verbindung etwa zwischen den Stücken Preambalon in la und Carmen in sol von Kleber (Kleber49, 11), Fantasie Nr. 3 für Laute von Luis Milán ${ }^{42}$ sowie Prooemium in re von Kotter (Kotter26) feststellen.

Diese Präludien sind durch vokal-tänzerisches motivisches Material charakterisiert. Besonders deutlich wird ein Zusammenhang von Präludien mit Vokal- und Tanzkompositionen in den Expositionsphasen von Werken, die das übernommene Material in strukturell abgeschlossene Abschnitte einbauen. Wichtig ist die Tendenz zur Einbeziehung von zwei Vokalwendungen oder von einer Vokalwendung und einer jener ähnlichen Variante in die Komposition (Judenkünig, Das fierd Priamel). Die Zunahme an kontrapunktischer Arbeit geht mit einer Abnahme von genuin instrumentalem Material wie Passagen und Akkorden Hand in Hand. Solche Präludien ähneln dem polyphonen Ricercar mit zwei Soggetti.

Jedoch versuchten die Komponisten das Präludium auch vor direkten Einflüssen von anderen Gattungen zu bewahren. Newsidler zum Beispiel ordnet seine zwei Preambeln aus Ein new künstlich Lautten Buch zwar in zwei verschiedene Rubriken ein - Ein gut Preambl für junge Schüler unter Deutsche Liedlein, Ein gut Preambl hingegen unter Welsche Tentz ${ }^{43}$-, die unterschiedliche Faktur erwarten lassen würden, schreibt jedoch in beiden Fällen eigenständige genuine Instrumentalpräludien mit dem typischen Kontrast von Passagen- und Akkordabschnitten [Notenbeispiel 15].

\footnotetext{
${ }^{42}$ Luis Milán, Libero de música de vihuela de mano. Intitulado El Maestro, hrsg. von Leo Schrade (= PÄM 2), Leipzig 1927.

43 Siehe Hans Gerle, Ein News sehr künstliches Lautenbuch 1552, Hans Newsidler, Das ander Buch 1549, Hans Newsidler, Das dritt Buch 1544.
} 

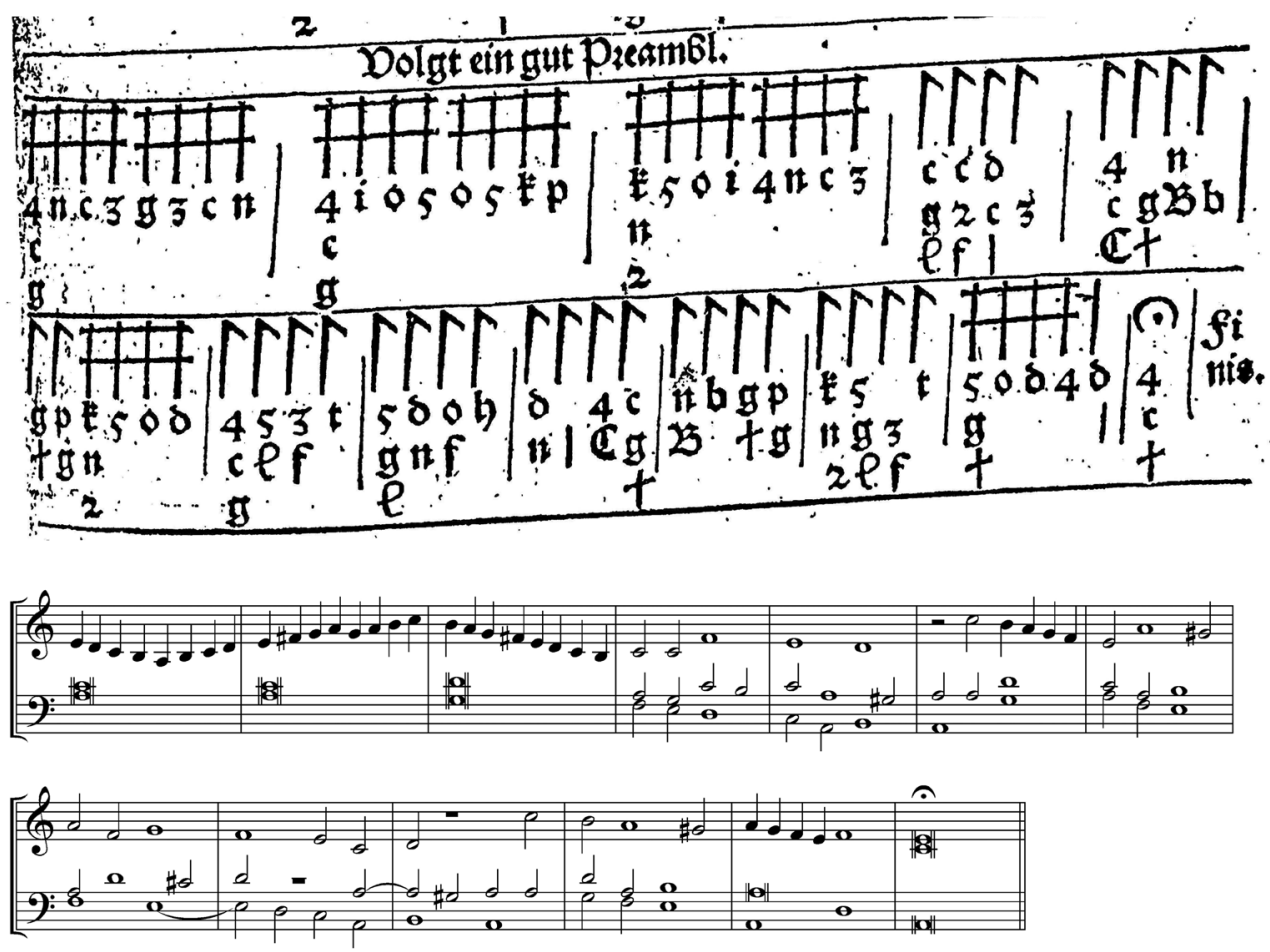

Notenbeispiel 154: Newsidler, „Ein gut Preambl“

$V$.

Alle Besonderheiten des deutsch-österreichischen Präludiums im 15. und 16. Jahrhundert lassen erkennen, dass es sich beim Präludium um ein offenes gattungsstilistisches System handelt. Dieses System ist eine für die Renaissancezeit spezifische Binnenform zwischen Gattungs- und Stilkonstrukten. Es ist durch eine Reihe von konstanten Gattungsmerkmalen charakterisiert und weist doch zugleich aufgrund bestimmter Kennzeichen allgemeine Stilmerkmale auf. Dadurch demonstriert dieses System Offenheit und Flexibilität.

Das Präludium als Gattung kennzeichnen folgende Merkmale: Es handelt sich um Instrumentalwerke, die durch pädagogische Zielsetzung und durch Fokussierung auf das Soloinstrumentalspiel (Orgel oder Laute) bestimmt sind. Aufgrund der didaktischen Aufgaben ist das Präludium keine freie Improvisation. Unmittelbar mit der Entwicklung des Instrumentalspiels und den daraus resultierenden didaktischen Aufgaben hängen Entstehung, Entwicklung und Periodisierung des Präludiums zusammen. Als Gattung entsteht das Präludium schon in Ileborghs Stücken; es entwickelt sich in zwei Richtungen weiter. In Abhängigkeit von den pädagogischen Zielen ist es als genuine Instrumentalform (vorwiegend in der Orgelmusik im 15. und 16. Jahrhundert und in der Lautenmusik bis zur Mitte des 16. Jahrhunderts) oder als Bearbeitung (vornehmlich in

${ }^{44}$ Diese Übertragung aus der Lautentabulatur wurde von mir angefertigt. 
der Lautenmusik der zweiten Hälfte des 16. Jahrhunderts) ausgeprägt. Jedoch basieren die Präludien immer auf einer ausgearbeiteten modal-harmonischen Form, die unabhängig vom Vorhandensein von Bezeichnungen „In Tono ..." ein bestimmendes Kriterium für das Präludium ist.

Mit anderen Kennzeichen überschreitet das Präludium als Gattung entweder die Stilebene oder es charakterisiert sich nur in Bezug auf konkrete Typen der Präludien. So betrifft die oft den Präludien zugeschriebene Einleitungsfunktion auch viele andere Gattungen in der Instrumentalmusik jener Zeit, vermag aber unter den Präludien selbst nur einer kleinen Gruppe von Stücken gerecht zu werden. Die in Präludien verwendeten melodisch-rhythmischen und teilweise auch modal-harmonischen Mittel und Formstrukturen sind auch anderen Gattungen nicht fremd, wenn sie auch in bestimmten Zusammenhängen verschiedene Typen von Präludien zu definieren vermögen.

Im vorliegenden Beitrag wurden zwei Präludientypen voneinander abgegrenzt. Auf der einen Seite stehen genuine Instrumentalpräludien, die entweder als Einleitung fungieren oder eine eigenständige Komposition repräsentieren. Auf der anderen Seite existieren Präludien, die Bearbeitungen von Vokal- und Instrumentalmusik darstellen und dabei entweder genau dem Vorbild folgen oder es variieren. Das Präludium mit Einleitungsfunktion weist homogenes Material (entweder Akkorde oder Passagen), rhythmisch freie, nicht in ein Taktschema einzuordnende Passagen und kleine Strukturen auf (ein- oder zweiteilige Perioden). Für das Präludium als eigenständige, nicht an ein nachfolgendes Stück gebundene Instrumentalform sind die Entwicklung eines Kontrastes zwischen den verwendeten Satztechniken (Akkorde - Passagen), die Verwendung akkordischer und passagenartiger Sequenzen, das Umspielen der Kadenzformeln und kompliziertere Formstrukturen (dreiteilige Periode, einteilige variierende Form, symmetrische und zweiteilige Formen) typisch. Für Präludien, die präzise nach einer Vorlage gearbeitet sind, ist die Originalquelle bekannt. Präludien-Bearbeitungen, in denen mit der Vorlage frei umgegangen wird, unterscheiden sich von den eigenständigen Präludien durch vokale und tänzerische Motivik, eine komplizierte imitatorisch-polyphone Technik und die Bevorzugung zweiteiliger Formen mit zwei ausgeprägten motivischen Ideen.

Alle genannten Besonderheiten zeichnen das Präludium als ein System aus und ermöglichen es, Abgrenzungen gegen verwandte Gattungen vorzunehmen, aber auch Zusammenhänge mit anderen Gattungen herzustellen. Ein akkordisches Gerüst unterscheidet das Präludium als Einleitung von der Kunstform Finale und von Stücken ohne Titel; das Fehlen imitatorischer Techniken kennzeichnet die Präludien des 15. Jahrhunderts gegenüber Redeuntes. Doch in Präludien des 15. Jahrhunderts sind auch schon die Ursprünge der Variationen über ein Harmoniegerüst erkennbar. Mit der Einbeziehung von Wechseln zwischen metrischer Zwei- und Dreiteiligkeit nähert sich das Präludium der Tanzmusik und den Fantasien des 16. Jahrhunderts. Die Entwicklung der imitatorischen Technik und die Verwendung von vokaler Motivik, von zwei- und mehrteiligen Formen mit Elementen kontrasthaft-zusammengesetzter Formen verbindet die Präludien dieser Zeit mit Ricercaren, Fantasien und Vokalbearbeitungen. Zugleich kündigen sich hier Vorformen der Verbindung von Präludium und Fuge an. 